Dieter Becker

Intangible Assets in der Unternehmenssteuerung 
Dieter Becker

\section{Intangible Assets in der Unternehmenssteuerung}

Wie Sie weiche Vermögenswerte quantifizieren und aktiv managen

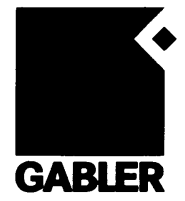


Bibliografische Information Der Deutschen Bibliothek

Die Deutsche Bibliothek verzeichnet diese Publikation in der Deutschen Nationalbibliografie;

detaillierte bibliografische Daten sind im Internet über <http://dnb.ddb.de> abrufbar.

Dieser Ausgabe liegt ein Post-it ${ }^{\circledR}$ Beileger der Firma

$3 \mathrm{M}$ Deutschland $\mathrm{GmbH}$ bei.

Wir bitten unsere Leserinnen und Leser um Beachtung.

1. Auflage 2005

Alle Rechte vorbehalten

(c) Betriebswirtschaftlicher Verlag Dr. Th. Gabler/GWV Fachverlage GmbH, Wiesbaden 2005

Lektorat: Jens Kreibaum

Der Gabler Verlag ist ein Unternehmen von Springer Science+Business Media.

www.gabler.de

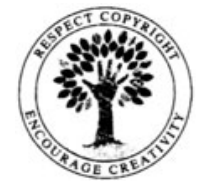

Das Werk einschließlich aller seiner Teile ist urheberrechtlich geschützt. Jede Verwertung außerhalb der engen Grenzen des Urheberrechtsgesetzes ist ohne Zustimmung des Verlags unzulässig und strafbar. Das gilt insbesondere für Vervielfältigungen, Übersetzungen, Mikroverfilmungen und die Einspeicherung und Verarbeitung in elektronischen Systemen.

Die Wiedergabe von Gebrauchsnamen, Handelsnamen, Warenbezeichnungen usw. in diesem Werk berechtigt auch ohne besondere Kennzeichnung nicht zu der Annahme, dass solche Namen im Sinne der Warenzeichen- und Markenschutz-Gesetzgebung als frei zu betrachten wären und daher von jedermann benutzt werden dürften.

Umschlaggestaltung: Nina Faber de.sign, Wiesbaden

Gedruckt auf säurefreiem und chlorfrei gebleichtem Papier 


\section{Inhaltsverzeichnis}

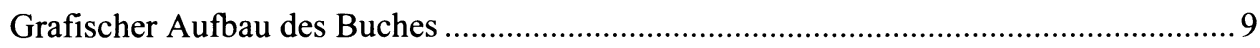

Geleitwort (Prof. Dr. Dirk Hachmeister, Universität Hohenheim, Lehrstuhl für Rechnungswesen und Finanzierung) ......................................................11

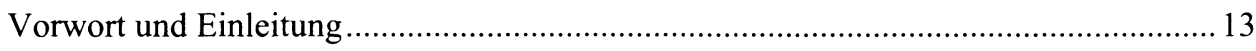

1. Ausgangssituation und Begriffsklärung........................................................... 17

1.1 Begriff der Intangible Values ....................................................................... 17

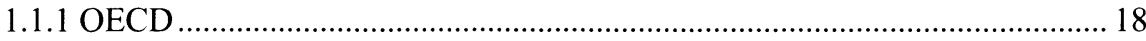

1.1.2 Arbeitskreis der Schmalenbach-Gesellschaft für

Betriebswirtschaft e.V............................................................................ 19

1.2 Berücksichtigung der externen Rechnungslegung …………………………….... 22

1.2.1 Immaterielle Werte nach HGB-Begrifflichkeiten ........................................... 24

1.2.2 Identifizierbare Immaterielle Vermögensgegenstände

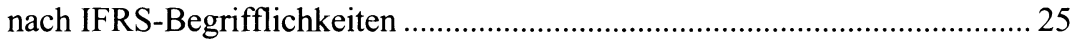

1.2.3 Identifizierbare Immaterielle Vermögensgegenstände nach US GAAP-Begrifflichkeiten .............................................................. 27

1.2.4 Geschäfts- oder Firmenwert/Goodwill

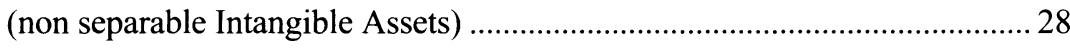

1.2.4.1 Geschäfts- oder Firmenwert nach HGB ............................................. 30

1.2.4.2 Goodwill nach IAS/IFRS .................................................................. 31

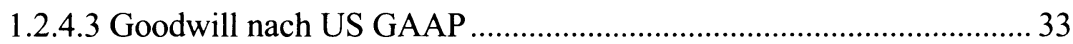

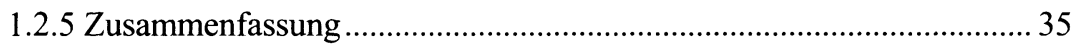

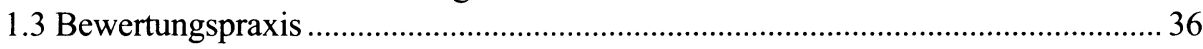

1.3.1 Monetäre Bewertung von Intangible Values.................................................... 36

1.3.2 Verfahren zur Darstellung und Bewertung immaterieller

Vermögensgegenstände ................................................................................ 40

1.3.2.1 Deduktiv summarische Ansätze.......................................................... 41

1.3.2.1.1 Market-to Book-Value ........................................................ 42

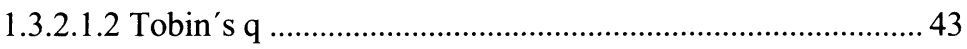

1.3.2.1.3 Calculated Intangible Value (CIV) ....................................... 45

1.3.2.1.4 Realoptionsansatz.................................................................... 46

1.3.2.2 Induktiv-analytische Ansätze: reine Bewertungsverfahren oder eigene Managementkonzepte? ................................................... 48

1.3.2.2.1 Kritische Würdigung der klassischen

Bewertungsmethoden am Beispiel „Kunde“........................ 49

1.3.2.2.2 Workonomics ${ }^{\mathrm{TM}}$, Custonomics ${ }^{\mathrm{TM}}$,

Suppliernomics $^{\mathrm{TM}}$......................................................... 51

1.3.2.3 Managementorientierte Bewertungsansätze ………………………....59

1.3.2.3.1 Intangible Assets Monitor (IAM) ................................................ 60

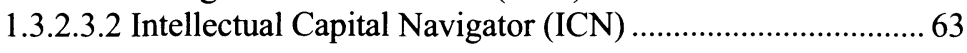


1.3.3 Zwischenfazit: Anforderungen an die Bewertung von Intangible Values auf der betrieblichen Ebene

2. Empirische Analyse DAX 30-Unternehmen 2000 - 2004 ……………………........ 73

2.1 Untersuchungsziel und -Untersuchungsmethodik ……………………………....... 73

2.2 Entwicklung der Aktiva im Zeitraum 2000 - 2004 ................................................. 75

2.3 Entwicklung der Immateriellen Vermögenswerte im Zeitraum 2000 - 2004.................................................................................... 78

2.4 Gesamtaktiva versus Immaterielle Vermögenswerte

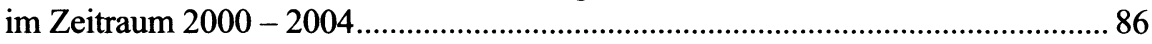

2.5 Entwicklung der Abschreibungen auf Immaterielle Vermögenswerte im Zeitraum 2000 - 2004 ......................................................... 89

2.6 Analyse der Intangible-Assets-Strategie der DAX 30-Unternehmen .......................96

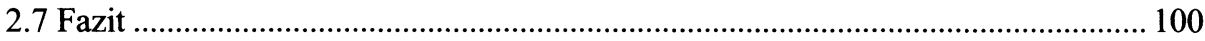

3. Managementkonzept für Intangible Values............................................................ 103

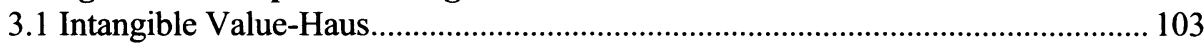

3.2 Die Vorgehensweise: Analyse von „best of" der bestehenden gesetzlichen Vorschriften, Bewertungs- und Managementkonzepte....................... 108

3.3 Das Konzept eines „Best of"-Verfahrens ……………………………………........ 112

3.4 Praxisbeispiel F\&E-Management ....................................................................... 115

3.4.1 Die innere Logik des Intangible Value-Hauses am Beispiel der F\&E

3.4.2 Systematik potenzieller Erfolgsfaktoren:

Sechs erfolgskritische Bausteine des F\&E-Prozesses .................................. 120

3.4.3 Erfolgslogik eines Unternehmens. Empirische Ergebnisse zu Erfolgsfaktoren im F\&E-Management

3.4.4 Empirische Ergebnisse der KPMG-Studie zu Erfolgsfaktoren im F\&E-Management

3.4.5 Aktuelle Tendenzen in der externen Rechnungslegung: Bilanzierung der F\&E-Aufwändungen gemäß IFRS

3.4.6 Bilanztechnische Synergieeffekte als Ergebnis des integrierten F\&E-Managements................................................................. 131

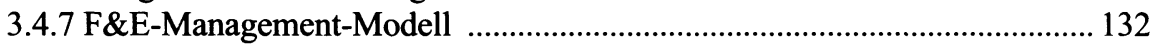

3.4.7.1 KPMG F\&E Performance Management Modell............................... 135

3.4.7.2 Strategisches F\&E-Management zahlt sich mehrmals aus

4. Praktische Relevanz des Human Capital Managements...................................143

4.1 Praktische Relevanz des HCM für die Bewertung der Unternehmensperformance durch Stakeholders ............................................... 145

4.1.1 Unternehmenskultur und Nachhaltigkeit ....................................................... 145

4.1.2 Interpretation der Nachhaltigkeit für das Human Capital ............................... 146 
4.1.3 Statische und dynamische Betrachtung der nachhaltigen

Unternehmensentwicklung und ihre Wirkungsweise auf das

Eigen- und das Fremdkapital.

4.1.4 Matrix der Human Capital-Instrumente

und Verbindung zur Nachhaltigkeit .......................................................... 151

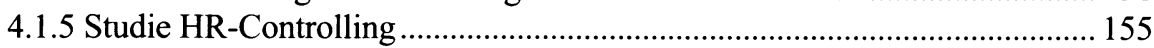

4.1.5.1 Durchführung in der Praxis ............................................................ 155

4.1.5.2 Ausrichtung des HR-Controlling auf ein explizites

Management des Human Capital....................................................... 159

4.1.5.3 Umsetzungsschwerpunkte............................................................... 163

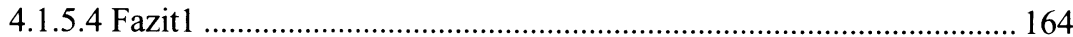

4.2 Praktische Relevanz des HCM für die Bewertung durch Kapitalmärkte und

Wirkungsweise der Marktbewertung auf das Investor Capital ............................. 165

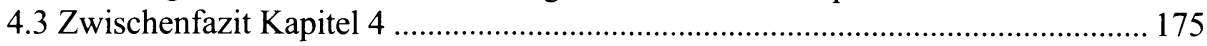

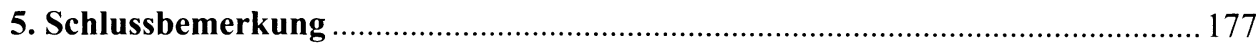

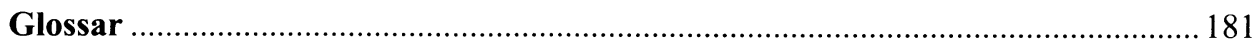

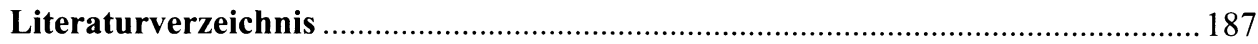

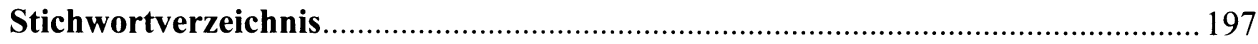

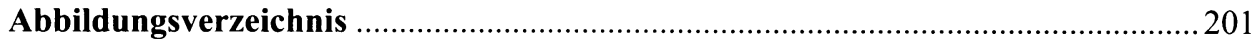

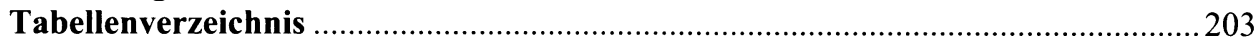

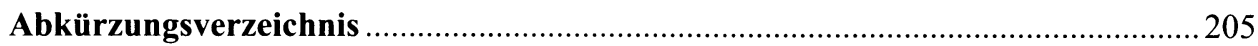

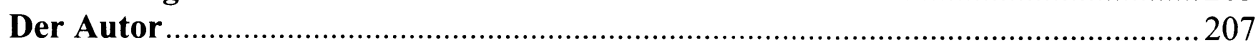




\section{Grafischer Aufbau des Buches}

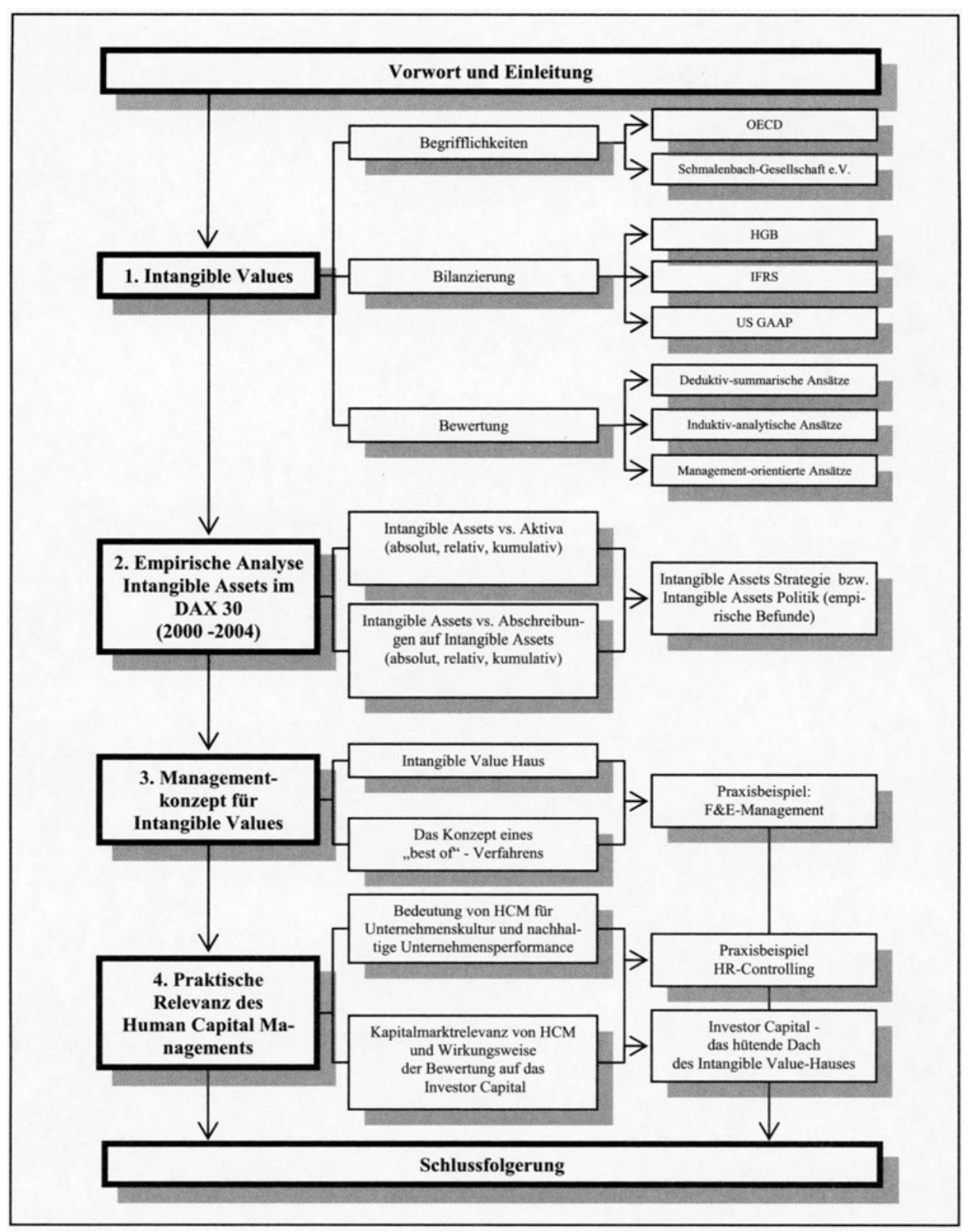




\section{Geleitwort}

Intangible Assets gehören zu den spannendsten Fragestellungen im Rahmen der Unternehmensführung, des Controllings und der Rechnungslegung. Leider werden sie jedoch wegen ihrer geringen „Greifbarkeit“ oftmals nur unzureichend in der Praxis beachtet. Zwar werden Fragen der Erfassung und Berichterstattung über immaterielle Vermögenswerte mittlerweile umfassend von Theorie und Praxis diskutiert, Fragen der Steuerung und Kontrolle von Intangible Assets werden hingegen sehr viel weniger beachtet. Dabei liegen hier die großen Herausforderungen für die Unternehmensführung und das Controlling.

Intangible Assets lassen sich im Gegensatz zu vielen materiellen Ressourcen nicht einfach über einen Markt beschaffen, sie müssen im Unternehmen geschaffen werden. Dies bedeutet aber auch, dass bei Intangible Assets unternehmensinterne Mechanismen die Erfolgsbeurteilung und Performancekontrolle übernehmen müssen, da der Preis als Maß des Erfolgs zumindest im Frühstadium der Entstehung von immateriellen Werten keine Relevanz hat. Hier stehen wir in Theorie und Praxis erst am Anfang der Entwicklung.

Das Ihnen vorliegende Buch von Dieter Becker liefert einen umfassenden Überblick über Ansätze der Messung und Beurteilung immaterieller Vermögenswerte, wie sie ihn nach meiner Einschätzung an anderer Stelle nicht finden. Über diese verdienstvolle Aufarbeitung bestehender Konzepte hinaus beschreitet seine Arbeit jedoch neue Wege: Zum einen bei der Beurteilung und Steuerung von Forschungs- und Entwicklungsprozessen, zum anderen beim Einsatz von Human Capital.

Spätestens in dem Moment, in dem Forschung und Entwicklung im Rechnungswesen nicht nur als Auszahlung, sondern als Leistung erfasst werden muss, wird deutlich, das eine Steuerung dieser Aktivitäten zwingend wird, weil bei Misserfolg in der Zukunft entsprechende Wertberichtigungen notwendig werden. Aber auch die Bedeutung des Human Capitals für den Unternehmenserfolg wird von Dieter Becker deutlich herausgestellt werden. Der Einsatz von ausreichend Human Capital zum richtigen Zeitpunkt bei der richtigen Fragestellung ist die Herausforderung, der sich die Unternehmen heute stellen müssen.

Ich bin mir sicher, dass dieses Buch als eine gelungene Mischung von theoretischen Konzepten und praktischer Erfahrung Ihnen konkrete Möglichkeiten zum Management von Intangible Assets aufzeigt. Ich wünsche Ihnen viel Spaß beim Lesen.

Prof. Dr. Dirk Hachmeister, Universität Hohenheim, Lehrstuhl für Rechnungswesen und Finanzierung 


\section{Vorwort und Einleitung}

"Shareholder Value ist out, Performance Measurement dagegen ist in."

Dies war die Einführung zu einem Buch mit dem Titel Value-Based Performance Management im Jahre 1999, an welchem ich selbst mitgeschrieben hatte. ${ }^{1}$ Beschrieben wurde damals die Herausforderung, die von den Eigentümern geforderte Wertorientierung in die interne Unternehmenssteuerung - und damit auf unterschiedliche Unternehmensebenen - zu übersetzen. Schon damals monierten die Autoren, dass die Herausforderung eines jeden finanziellen Steuerungsmodells darin liege, seine betriebswirtschaftlich sinnvollen Grundsätze in die eigentliche Unternehmenssteuerung zu übertragen.

Für die Zeit seit 1999 kann festgehalten werden, dass es einer großen Anzahl der Unternehmen gelungen ist, die verschiedenen Steuerungsebenen des Unternehmens miteinander zu verknüpfen und somit eine Durchgängigkeit der finanziellen Steuerungsprozesse in der Organisation erreicht zu haben.

Ist somit das gesetzte Postulat in der Zwischenzeit erreicht worden?

Damals wurde ebenfalls bereits im Vorwort gefordert: „Dabei dürfen Unternehmen jedoch nicht nur einseitig und kurzfristig Optimierungen im Sinne des Shareholder Value anstreben, sondern müssen vielmehr ihre Kompetenzen und Fähigkeiten auf die neuen technologischen und marktbezogenen Entwicklungschancen ausrichten“.

Wie wir in der Zwischenzeit wissen, ist genau das Gegenteil dieses Postulates eingetreten: Viele Unternehmen haben die Forderung nach nachhaltiger Wertsteigerung ganz deutlich mit der Forderung nach kurzfristiger Ergebnissteigerung verwechselt. Themen wie Kompetenzen und Fähigkeiten der Mitarbeiter, flexible Strukturen und sich selbst steuernde Prozesse, leistungsfähige Aufbau- und Ablauforganisation stehen heute im Mittelpunkt der fachlichen sowie beratenden Diskussion. Dennoch werden die in diese Termini gesetzten Erwartungen auf der betrieblichen Ebene oftmals nicht realisiert. Leider lassen sich die Managementbemühungen in finanziellen Kennzahlen nicht oder nicht im gewünschten Maße wieder finden. Offensichtlich liegt die Ursache einer solchen Zielverfehlung in einem nicht ausgefeilten Mess- und Anreizsystem, da viele Unternehmensführungen die einseitige (und auch eigene) finanzielle Optimierung in den Vordergrund gestellt haben. Dies liegt natürlich auch daran, dass finanzieller Erfolg auch am einfachsten messbar ist.

„You simply can't manage anything you can't measure. “2

\footnotetext{
${ }^{1}$ Brunner, J./Becker, D./Bühler, M./Hildebrandt, J./Zaich, R., Value-Based Performance Management. Wertsteigernde Unternehmensführung: Strategie, Instrumente, Praxisbeispiele, Wiesbaden 1999

${ }^{2}$ Quinn (1997), Vice President Quality, Sears Merchandising Group
} 
Diese Aussage aus der Einleitung ist die wesentliche Ursache der aktuell zu beobachtenden Fehlentwicklung und leitet über in die Themenstellung, welche Inhalt dieses Buches sein wird. Denn "manchmal kann das, was zählt, nicht gezählt werden, und das, was gezählt werden kann, zählt nicht" ${ }^{\prime 3}$. So sind Kompetenzen, Fähigkeiten, Aufbau- und Ablaufstrukturen Themenstellungen, welche nicht einfach messbar sind. Das Buch versucht, eine Erfassung und Gliederung all dieser eher nicht materiellen und schwer messbaren Werte vorzunehmen, um an verschiedenen Beispielen aufzuzeigen, dass Ursache eines finanziellen Erfolges im Zeitablauf exakt das Management dieser Werte ist.

„In den Jahresabschlüssen taucht das, intellektuelle Vermögen' der Unternehmen bislang nicht auf, weil es sich nicht in Euro messen lässt. Banken und Anleger aber werden künftig mehr Einblick in die wahren Werte verlangen... "“

Ziel dieses Buches soll jedoch nicht sein, ein exaktes Mess- und Steuerungsinstrumentarium für nicht-materielle Werte zu entwickeln, sondern vielmehr mithilfe von Ursache-Wirkungsketten die Aufmerksamkeit der Unternehmensführung auch auf diese Werte zu lenken.

Motto dieses Buches soll ein Zitat von Johann Wolfgang von Goethe sein:

„Das erste Anzeichen, dass wir nicht wissen, was wir tun, ist eine Besessenheit von Zahlen.“

Es wird viel über Intangible Values gesprochen, den Eingang in das tägliche Management hat aber dieser Begriff bis dato noch nicht gefunden. Verwendet man einen solchen Begriff, so muss zunächst eine umfassende Abgrenzung erfolgen. Diese Abgrenzung umfasst sowohl den Begriff als solchen als auch die gängige Bilanzierungsund Bewertungs- und auch Steuerungspraxis sowie eine Kategorisierung.

Zur inhaltlichen Fundierung wird eine Analyse der Intangible Assets der Bilanzen der DAX 30-Unternehmen für die Jahre 2000 bis 2004 herangezogen. Aus dieser Analyse kann der Umgang mit aktivierten immateriellen Werten der DAX 30Unternehmen im Rahmen der externen Berichterstattung (gemäß HGB und/oder IFRS und/oder US-GAAP) nachvollzogen werden. Zum einen wird erkennbar sein, dass trotz der heftigen Diskussion zum Thema Abschreibungen auf immaterielle Werte den jeweiligen Jahresabschlüssen nicht viel über die Strategien zum Schaffen, Erhalten und Weiterausbauen der immateriellen Ressourcen entnommen werden kann. Zum anderen kann man aber generell feststellen, dass, bereits in der Begriffsabklärung, welche sich in Firmen(Lage-)berichten sowie in zahlreichen Fachveröffentlichungen finden lässt, eine Bedeutung der Intangible Assets für den zukünftigen Unternehmenserfolg erkennbar geworden ist.

\footnotetext{
${ }^{3}$ Physiker und Nobelpreisträger Albert Einstein (1879 - 1955)

${ }^{4}$ Financial Times Deutschland, 07.09.2004, S. 29
} 
Als Conclusio der ersten beiden Teile kann festgehalten werden, dass es zwar viele Ansätze zur Begriffsbestimmung gibt, die einzelnen Begriffe aber weder in einen inhaltlichen noch zeitlichen Zusammenhang gesetzt wurden.

Stehen diese Begriffe tatsächlich nur nebeneinander oder lässt sich ein Modell eines Intangible Value-Hauses ableiten, welches über bestimmte Fundamente, Rahmenbedingungen und Erfolgsfaktoren verfügt? Zielsetzung des dritten Kapitels ist die Ableitung eines Modells, welches sowohl inhaltliche Ursache-Wirkungszusammenhänge aufzeigt, als auch zeitliche Abfolgen sicherstellt. Somit werden zunächst Begriffsklärungen durchgeführt und einzelne Elemente erläutert, um anschließend ein Ursache-Wirkungsmodell in zeitlicher Abfolge darzustellen.

Neben diesem Ursache-Wirkungsmodell geht es aber im dritten Kapitel auch um ein Konzept, nach welchen Schritten ein erfolgreiches Management von Intangible Assets vorgenommen werden kann. Um es schon vorwegzunehmen: Dieses Modell wird verschiedene Elemente aus der externen Rechnungslegung und der internen Berichterstattung kombinieren und in eine sinnvolle Abfolge bringen.

All diese Modelle wären wirkungslos und theoretisch, wenn sie nicht in einen konkreten Zusammenhang gebracht werden und an einem praxisnahen Beispiel erläutert werden könnten. Aus diesem Grund wird in diesem Teil des Buches das Modell an der Thematik des F\&E-Managements praxisnah erläutert.

Ausgehend von dem oben beschriebenen Modell wird nun in einem nächsten Teil das Fundament, nämlich das Human Capital in den Mittelpunkt gestellt. Bci dem Modell hat ein Faktor, nämlich die Frage der Unternehmenskultur und der Nachhaltigkeit, besondere Bedeutung. Am praktischen Beispiel des Human Capital-Managements wird aufgezeigt, mit welchen Instrumenten die Unternehmen heute steuern, und in welchem Maße die Elemente des vorgeschlagenen Modells berücksichtigt werden. Neben der Begriffsabklärung geht es hier um Praxisbeispiele aus dem HCManagement. Erkennbar wird in diesem Kapitel, dass das HC als Basisfaktor in der letzten Zeit an Bedeutung zugenommen hat. Dennoch stecken die HC-Steuerungsinstrumente in den Kinderschuhen, da sich diese Instrumente sehr singulär mit HC beschäftigen. Es wird oft übersehen oder nicht ausreichend beachtet, dass der langfristige Unternehmenserfolg durch komplexe Ursache-Wirkungsketten der HCSteuerung zu den anderen materiellen sowie immateriellen (Erfolgs-)Faktoren wesentlich beeinflusst wird, wenn nicht gar davon abhängt.

Dieses Buch soll dazu dienen, der Bedeutung des immateriellen Vermögens im Unternehmen einerseits mehr Aufmerksamkeit zu verschaffen und andererseits Hinweise auf gut nutzbare Methoden und Verfahren zu geben, die in vielen Unternehmen schon Anwendung finden könnten. Die Problematik liegt damit weniger in dem mangelnden Vorhandensein von Methoden und Instrumenten, sondern vielmehr in der mangelnden organisatorischen Verankerung in den Unternehmen, was nicht zuletzt auch daran liegt, dass durch die funktionale Trennung von Strategischem und 
Operativem Controlling und Externem Rechnungswesen ein Austausch in vielen Unternehmen nicht mehr praktiziert wird.

Das Buch richtet sich einerseits an Führungskräfte in Unternehmen, die sich einen ersten Überblick der gängigen Verfahren und Methoden verschaffen wollen, und andererseits an Menschen, die realisiert haben, dass die eigentliche Wertschaffung in Unternehmen in einem erfolgreichen Management des immateriellen Vermögens begründet liegt.

Das Konzept und die Umsetzung dieses Buches ist über die vergangenen zwei Jahre durch intensive Diskussionen mit Freunden und Kollegen entstanden. Ziel war es, stets einen notwendigen Kompromiss zwischen Aktualität und wissenschaftlichem Anspruch zu erreichen.

Herausheben möchte ich die Diskussionen mit Rainer J. Schaetzle, HR-Direktor der Allianz Versicherungs AG. Mit Ihm habe ich von Beginn an intensiv und auch kontrovers über das Thema diskutieren dürfen. Er hat mir wichtige Impulse und Denkanstösse insbesondere zu Kapitel 4 gegeben.

Ich danke Bernd Schmid, Vorstand der KPMG Deutsche Treuhand Gesellschaft AG, für die Förderung dieses Projektes. Ohne diese Förderung wäre eine Realisierung neben der Vielzahl von umfangreichen anderen Projektarbeiten nie möglich gewesen.

Insbesondere Olga Chaikevitch bin ich für die zahlreichen inhaltlichen Hinweise und Hilfestellungen neben dem unermüdlichen Korrektureinsatz sehr zu Dank verpflichtet.

Armin Steiner, Andreia Guedes Campos und Cinzia Lazzari haben mit unermüdlichem Einsatz an Änderungen und Korrekturen gearbeitet. Auch Ihnen einen herzlichen Dank.

Dieter Becker 